\title{
Useful Web sites for researchers studying proteins
}

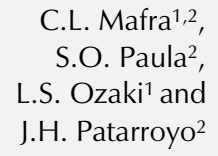

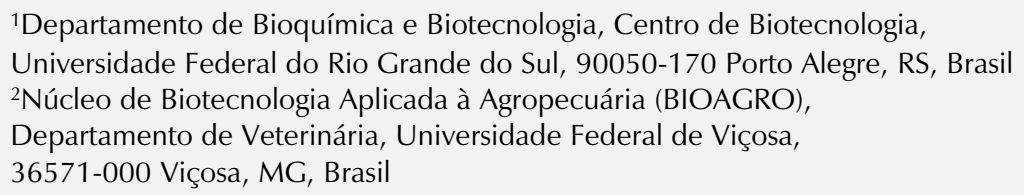

'Departamento de Bioquímica e Biotecnologia, Centro de Biotecnologia, Universidade Federal do Rio Grande do Sul, 90050-170 Porto Alegre, RS, Brasil ${ }^{2}$ Núcleo de Biotecnologia Aplicada à Agropecuária (BIOAGRO), Departamento de Veterinária, Universidade Federal de Viçosa, 36571-000 Viçosa, MG, Brasil

\section{Correspondence}

C.L. Mafra

Núcleo de Biotecnologia Aplicada

à Agropecuária (BIOAGRO)

Departamento de Veterinária

Universidade Federal de Viçosa

Av. P.H. Rolfs, s/n

36571-000 Viçosa, MG

Brasil

E-mail: sctmafra@mail.ufv.br

Research supported by CAPES. C.L. Mafra is the recipient of a predoctoral fellowship from CAPES.

Received November 27, 1996 Accepted January 13, 1997

\section{Introduction}

The development of high performance computers both at hardware and software levels associated with the exponential growth of the Internet has opened new sources of information in all areas of knowledge (1). For biologists, the World Wide Web (WWW) today offers alternative information sources which are much easier to reach and unthinkable only a few years ago $(2,3)$. In this Web, it is possible, for instance, to access libraries and databases, to engage in courses and discussion groups, and to use programs online (4), far beyond the electronic mail (email), file transference protocol (ftp) and document retrieval system of the old days. Presently, technologies are explosively being developed that will enable direct visual contact world wide, both personal and in conference, and online discussion of files and data. Programs already exist whose better performance still depends on the development of the Internet itself.

In the present minireview we list the many Uniform Resource Locators (URLs) organized in different areas such as Immunology, Chemistry, Biochemistry, Molecular Biology and Crystallography, all of common interest to researchers dealing with protein-related subjects. Similar lists were compiled for cell and tissue culture (5), human genetics (6), molecular biology (7), thermocycling (8) and biotechnology companies (9).

We organized the Web pages by subjects related to protein research (Table 1), tools for online protein analysis (Table 2), organizations with useful references (Table 3), pages with announcements of meetings and related courses (Table 4), scientific journals (Table 5), and some biotechnology companies commonly consulted by the community (Table 6).

As the Web continually expands, this directory does not intend by any means to be complete. We hope, however, that it will be helpful as a starting point to look for and find useful protein-related information in the Web.
Key words

- Protein analysis

- WWW

- Resource 
Table 1 - Protein-related sites.

\begin{tabular}{|c|c|}
\hline & URL \\
\hline \multicolumn{2}{|l|}{ Biochemistry } \\
\hline Links to bioinformatics center-related servers & http://www.biochem.ucl.ac.uk/bsm/dbbrowser/sites.html \\
\hline Related protein structure links & http://www.yorvic.york.ac.uk/computing/links.html \\
\hline Web resources for protein scientists & http://www.prosci.uci.edu/ProSciDocs/WWWResources.html \\
\hline $\begin{array}{l}\text { WWW Virtual library: Biochemistry and } \\
\text { molecular biology }\end{array}$ & http://golgi.harvard.edu/biopages/biochem.html \\
\hline WWW Virtual library: Biomolecules & http://golgi.harvard.edu/sequences.html \\
\hline \multicolumn{2}{|l|}{ Biotechnology } \\
\hline BioTech Science resources & http://biotech.chem.indiana.edu/pages/scitools.html \\
\hline Pedro's biomolecular research tools & http://www.public.iastate.edu/ pedro/research_tools.html \\
\hline Useful links from Darst lab & http://darst-sgi2.rockefeller.edu/ \\
\hline WWW Virtual library: Biotechnology & http://www.cato.com/interweb/cato/biotech/ \\
\hline \multicolumn{2}{|l|}{ Chemistry } \\
\hline Chemistry databases and data collections & http://www.rpi.edu/dept/chem/cheminfo/chemres/chemres_03.html \\
\hline Chemistry Today - Jobs, news and meetings & http://chemistry.mond.org/ \\
\hline Internet chemistry index & http://www.chemie.fu-berlin.de/chemistry/index/ \\
\hline Internet chemistry resources & http://www.rpi.edu/dept/chem/cheminfo/chemres.html \\
\hline Latinamerican Web of chemistry & http://latina.chem.cinvestav.mx/ \\
\hline Molecular simulation on the Web & http://ganter.chemie.uni-dortmund.de/comp_chem.html \\
\hline $\begin{array}{l}\text { Silicon Grafics - Chemistry and biological } \\
\text { sciences }\end{array}$ & http://www.sgi.com/ChemBio/ \\
\hline $\begin{array}{l}\text { WWW Chemistry resources, jobs and academic } \\
\text { and commercial sites }\end{array}$ & http://www-chem.harvard.edu/webchemistry/webchem.html \\
\hline WWW Computational chemistry resources & http://www.chem.swin.edu.au/chem_ref.html \\
\hline \multicolumn{2}{|l|}{ Immunology } \\
\hline Antibody resources & $\begin{array}{l}\text { http://www- } \\
\text { chem.ucsd.edu/Faculty/goodman/antibody.html/abpage.html }\end{array}$ \\
\hline Antibody structure and sequence information & http://www.biochem.ucl.ac.uk/ martin/antibodies.html \\
\hline Links to cytokine-related sites & http://www.lmb.uni-muenchen.de/groups/ibelgaufts/links.html \\
\hline $\begin{array}{l}\text { Kabat database - Sequences of proteins of } \\
\text { immunological interest }\end{array}$ & http://immuno.bme.nwu.edu/ \\
\hline WWW Virtual library: Immunology & http://golgi.harvard.edu/biopages/immuno.html \\
\hline \multicolumn{2}{|l|}{ Molecular Biology } \\
\hline Biologists's control panel & http://gc.bcm.tmc.edu:8088/bio/bio_home.html \\
\hline DNA vaccine Web & http://www.genweb.com/Dnavax/dnavax.html \\
\hline $\begin{array}{l}\text { Compilation of resources in Internet for the } \\
\text { molecular biologist }\end{array}$ & http://www.ifrn.bbsrc.ac.uk/gm/lab/docs/iftmb.html \\
\hline Molecular biology information servers & http://research.nwfsc.noaa.gov/bioinformatics.html \\
\hline Molecular biology protocols & $\begin{array}{l}\text { http://grimwade.biochem.unimelb.edu.au/sigtrans.htm } \\
\text { http://research.nwfsc.noaa.gov/protocols.html }\end{array}$ \\
\hline Molecular biology related WWW sites & http://www.oxmol.co.uk/oth_link.html \\
\hline $\begin{array}{l}\text { Molecular biology servers, databases and Web } \\
\text { sites }\end{array}$ & http://pdb.pdb.bnl.gov/mole.html \\
\hline The Biocatalog in molecular biology and genetics & http://www.ebi.ac.uk/biocat/ \\
\hline
\end{tabular}


Table 2 - Analysis and search online.

\begin{tabular}{|c|c|c|c|}
\hline Institution & Service & Mnemonic & URL \\
\hline BMERC & Protein sequence analysis & & http://bmerc-www.bu.edu/protein-seq/ \\
\hline $\mathrm{BCM}$ & Protein secondary structure prediction & & http://dot.imgen.bcm.tmc.edu:9331/pssprediction/pssp.html \\
\hline $\begin{array}{l}\text { Center for } \\
\text { Molecular } \\
\text { Modeling - NIH }\end{array}$ & Protein database and analyzed sequences & & http://cmm.info.nih.gov/modeling/net_services.html \\
\hline \multirow[t]{2}{*}{ CBS } & O-glycosylation sites in mammalian proteins & NetOglyc & http://www.cbs.dtu.dk/netOglyc/cbsnetOglyc.html \\
\hline & $\begin{array}{l}\text { Signal peptide cleavage sites in amino acid } \\
\text { sequences }\end{array}$ & Signalp & http://www.cbs.dtu.dk/signalp/cbssignalp.html \\
\hline CNRS & $\begin{array}{l}\text { Assistance with the analysis of domain } \\
\text { arrangement in proteins }\end{array}$ & ProDom & http://protein.toulouse.inra.fr/prodom/form.html \\
\hline CNUSC & Immunogenetic database & IMGT & http://imgt.cnusc.fr:8104 \\
\hline Cohen Group & Protein secondary structure prediction & NNPREDICT & http://www.cmpharm.ucsf.edu/ nomi/nnpredict.html \\
\hline CSBS & Structural classification of proteins & SCOP & http://scop.mrc-lmb.cam.ac.uk/scop/ \\
\hline \multirow[t]{2}{*}{ EBI } & Protein database search & BLITZ & http://www.ebi.ac.uk/searches/blitz_input.html \\
\hline & Nucleotide to protein translation & $\begin{array}{l}\text { The Protein } \\
\text { Machine }\end{array}$ & http://www.ebi.ac.uk/contrib/tommaso/translate.html \\
\hline \multirow[t]{6}{*}{ EMBL } & $\begin{array}{l}\text { Protein secondary structure prediction from } \\
\text { a single sequence or from a set of sequences }\end{array}$ & PREDATOR & http://www.embl-heidelberg.de/argos/predator/predator_form.html \\
\hline & $\begin{array}{l}\text { Search for multiple sequence alignments, } \\
\text { prediction of secondary structure, solvent } \\
\text { accessibility, transmembrane helices, } \\
\text { topology for transmembrane protein, fold } \\
\text { recognition by prediction-based threading, } \\
\text { evaluation of secondary structure prediction } \\
\text { accuracy }\end{array}$ & PredictProtein & http://www.embl-heidelberg.de/predictprotein/predictprotein.html \\
\hline & Search for structural similarity & PROPSEARCH & http://www.embl-heidelberg.de/prs.html \\
\hline & Database of secondary structure assignments & DSSP & http://www.embl-heidelberg.de:80/srs/srsc?-info+DSSP \\
\hline & Secondary structure prediction of proteins & SSPRED & http://www.embl-heidelberg.de/sspred/sspred_info.html \\
\hline & Transmembrane segments prediction & TMAP & http://www.embl-heidelberg.de/tmap/tmap_sin.html \\
\hline \multirow[t]{5}{*}{ ExPASy } & $\begin{array}{l}\text { Theoretical } \mathrm{pI} \text { and } \mathrm{MW} \text { for a protein } \\
\text { sequence }\end{array}$ & $\mathrm{pI} / \mathrm{MW}$ & http://expasy.hcuge.ch/ch2d/pi_tool.html \\
\hline & Enzyme nomenclature database & & http://expasy.hcuge.ch/sprot/enzyme.html \\
\hline & $\begin{array}{l}\text { Physical and chemical parameters of protein } \\
\text { sequences }\end{array}$ & ProtParam & http://expasy.hcuge.ch/sprot/protparam.html \\
\hline & $\begin{array}{l}\text { Compute and represent the profile produced } \\
\text { by any amino acid sequence } \\
\text { (hydrophobicity, polarity, MW, secondary } \\
\text { structure prediction, etc) }\end{array}$ & ProtScale & http://expasy.hcuge.ch/cgi-bin/protscale.pl \\
\hline & $\begin{array}{l}\text { Automated knowledge-based protein } \\
\text { modeling server }\end{array}$ & Swiss-Model & http://expasy.hcuge.ch/swissmod/SWISS-MODEL.html \\
\hline FU Berlin & Amino acid structure & & http://www.chemie.fu-berlin.de/chemistry/bio/aminoacids.html \\
\hline IBCP & $\begin{array}{l}\text { Sequence analysis, secondary structure } \\
\text { prediction and pattern search in protein } \\
\text { database }\end{array}$ & & http://www.ibcp.fr/mailserver.html \\
\hline \multirow[t]{2}{*}{ ISREC } & Statistical Analysis of Protein Sequence & SAPS & http://ulrec3.unil.ch/software/SAPS_form.html \\
\hline & $\begin{array}{l}\text { Prediction of transmembrane regions and } \\
\text { orientation }\end{array}$ & TMpred & http://ulrec3.unil.ch/software/TMPRED_form.html \\
\hline $\begin{array}{l}\text { Johns Hopkins } \\
\text { University } \\
\text { Bioinformatics } \\
\text { Web Server }\end{array}$ & $\begin{array}{l}\text { Searching for software and data for biology } \\
\text { and general, available on the Internet }\end{array}$ & & http://www.gdb.org/Dan/softsearch/softsearch.html \\
\hline MRC/HGMP & $\begin{array}{l}\text { Protein sequence databases menu - A } \\
\text { collection of protein sequence databases, } \\
\text { motifs and protein families and some } \\
\text { information on 3D structures }\end{array}$ & & http://www.hgmp.mrc.ac.uk/Public/protein-seq-db.html \\
\hline NCBI & GenBank database & & http://www2.ncbi.nlm.nih.gov/genbank/query_form.html \\
\hline $\mathrm{NIH}$ & Three-dimensional drug structure databank & & http://cmm.info.nih.gov/modeling/drugbank.html \\
\hline
\end{tabular}


Table 2 - Analysis and search online (continued).

\begin{tabular}{|l|l|l|l|}
\hline Institution & Service & Mnemonic & URL \\
\hline NIBB & $\begin{array}{l}\text { Analysis and prediction of protein sorting } \\
\text { signals coded in amino acid sequence }\end{array}$ & PSORT & http://psort.nibb.ac.jp/ \\
\hline SBDS & Secondary structure prediction & GOR & http://molbiol.soton.ac.uk/compute/GOR.html \\
\hline $\begin{array}{l}\text { Skirball Institute } \\
\text { Medicine }\end{array}$ & Amino acid images & ftp://128.122.10.5/pub/pmsa/images & \\
\cline { 2 - 4 } & Amino acid properties & BioSCAN & http://genome.cs.unc.edu/online.html \\
$\begin{array}{l}\text { University of } \\
\text { North Carolina at } \\
\text { Chapel Hill }\end{array}$ & Biosequence analysis & & \\
\hline
\end{tabular}

\section{Table 3 - Reference organizations.}

\begin{tabular}{|c|c|c|}
\hline Organization & Abbreviation & URL \\
\hline American Association for the Advancement of Science & AAAS & http://www.aaas.org/ \\
\hline American Chemical Society & ACS & http://www.acs.org/ \\
\hline American Type Culture Collection & ATCC & http://www.atcc.org/atcc.html \\
\hline Argos Group & & http://www.embl-heidelberg.de/ExternalInfo/argos/argos-group.html \\
\hline Baylor College of Medicine & $\mathrm{BCM}$ & http://www.bcm.tmc.edu/ \\
\hline BioMolecular Engineering Research Center & BMERC & http://bmerc-www.bu.edu/bmerc-main.html \\
\hline Birkbeck College - Department of Crystallography & & http:/www.cryst.bbk.ac.uk/ \\
\hline Brookhaven National Laboratory & $\mathrm{BNL}$ & http://www.bnl.gov/ \\
\hline Cambridge School of Biological Sciences & CSBS & http://www.bio.cam.ac.uk/ \\
\hline Cambridge University Chemical Laboratory & & http://www.ch.cam.ac.uk/ \\
\hline Center for Biological Sequence Analyses & CBS & http://www.cbs.dtu.dk/ \\
\hline Centre de Recherche en Biochimie Macromoleculaire & & http://xerxes.crbm.cnrs-mop.fr/ \\
\hline Centre National de la Recherche Scientifique & CNRS & http://www.cnrs.fr/ \\
\hline Centre National Universitaire Sud de Calcul & CNUSC & http://www.cnusc.fr/ \\
\hline Cohen Group & & http://www.cmpharm.ucsf.edu/cohen/ \\
\hline European Bioinformatics Institute & EBI & http://www.ebi.ac.uk/ebi_home.html \\
\hline European Collection of Animal Cell Cultures & ECACC & http://www.gdb.org/annex/ecacc/HTMLecacc1.html \\
\hline European Molecular Biology Laboratory & EMBL & http://www.embl-heidelberg.de/ \\
\hline European Molecular Biology Network & EMBnet & http://beta.embnet.unibas.ch/embnet/info.html \\
\hline Expasy - Molecular Biology Server & ExPASy & http://expasy.hcuge.ch/ \\
\hline Free University Berlin - Department of Chemistry & FU Berlin & http://www.chemie.fu-berlin.de/index_e.html \\
\hline Food and Drug Administration & FDA & http://www.fda.gov/ \\
\hline Harvard Biological Laboratories - Genome Research & & http://golgi.harvard.edu/ \\
\hline Institute of Biology and Chemistry of Proteins & $\mathrm{IBCP}$ & http://www.ibcp.fr/ \\
\hline Medical Research Council & MRC & http://www.mrc.ac.uk/MRC/ \\
\hline National Academy of Sciences & NAS & http://www.nas.edu/ \\
\hline National Center for Biotechnology Information & NCBI & http://www.ncbi.nlm.nih.gov/ \\
\hline National Institute for Basic Biology & NIBB & http://www.nibb.ac.jp/ \\
\hline National Institutes of Health & $\mathrm{NIH}$ & http://www.nih.gov/ \\
\hline National Science Foundation & NSF & http://www.nsf.gov/ \\
\hline Northwest Fisheries Science Center & NWFSC & http://research.nwfsc.noaa.gov/ \\
\hline Oxford Molecular Group & & http://www.oxmol.co.uk/ \\
\hline Protein Data Bank & PDB & http://www.pdb.bnl.gov/ \\
\hline Shouthampton Bioinformatics Data Server & & http://molbiol.soton.ac.uk/sbds.html \\
\hline Skirball Institute of Biomolecular Medicine & & http://www.med.nyu.edu/Skirball/HomePage.html \\
\hline Swiss Institute for Experimental Cancer Research & ISREC & http://www-isrec.unil.ch/isrec.htm \\
\hline Weizmann Institute & & http://www.weizmann.ac.il/ \\
\hline
\end{tabular}


Table 4 - Meetings, courses and workshops on protein and related areas.

\begin{tabular}{|l|l|l|}
\hline Announcer & Comments & URL \\
\hline $\begin{array}{l}\text { American Chemistry } \\
\text { Society Meetings }\end{array}$ & $\begin{array}{l}\text { A list of meetings in chemistry } \\
\text { by geographical areas in USA }\end{array}$ & http://www.acs.org/meetings/welcome.htm \\
\hline Bioinformatics Courses & $\begin{array}{l}\text { A list of bioinformatics courses } \\
\text { in USA, UK and Europe }\end{array}$ & http://www.biochem.ucl.ac.uk/bsm/dbbrowser/courses.html \\
\hline Bioinformatics Practical & $\begin{array}{l}\text { A pratical guide on protein } \\
\text { sequence and structure analysis } \\
\text { on line }\end{array}$ & http://www.biochem.ucl.ac.uk/bsm/dbbrowser/jj \\
\hline $\begin{array}{l}\text { Cato Research's List of } \\
\text { Meetings }\end{array}$ & $\begin{array}{l}\text { A list of meetings in biotech- } \\
\text { nology and pharmaceutical } \\
\text { development around the world }\end{array}$ & http://www.catosource.com/fyi \\
\hline $\begin{array}{l}\text { Calendar of Events - Center } \\
\text { for Molecular Modeling/ } \\
\text { NIH }\end{array}$ & $\begin{array}{l}\text { Events in chemistry, biology } \\
\text { and related subjects }\end{array}$ & http://cmm.info.nih.gov/modeling/events.html \\
\hline Chemical Conferences & $\begin{array}{l}\text { Conferences on the various } \\
\text { disciplines of chemistry }\end{array}$ & http://hackberry.chem.niu.edu:70/0/ConferenceListings/SpecialistMeetings/index.html \\
http://ccl.osc.edu/ccl/crc.html
\end{tabular}

\section{Table 5 - Publishers and scientific journals.}

\begin{tabular}{|l|l|}
\hline Publisher or Journal & URL \\
\hline Academic Press Journals & http://www.apnet.com/www/journal/journals.htm \\
\hline American Chemistry Society Publications & http://pubs.acs.org/ \\
\hline Biochemistry and Molecular Biology Journals & http://www.princeton.edu/ beasley/journals.html \\
\hline Blackwell Science & http://www.blacksci.co.uk/ \\
\hline Cambridge University Press & http://www.cup.cam.ac.uk/ \\
\hline Cell & http://www.cell.com/ \\
\hline Cold Spring Harbor Laboratory Press & http://www.cshl.org/admin/press.html \\
\hline CRC Press, Inc. & http://www.crcpress.com/ \\
\hline CW Henderson & http://www.homepage.holowww.com/ \\
\hline Elsevier Science & http://www.elsevier.nl:80/ \\
\hline Gene & http://www.elsevier.com:80/catalogue/SAH/185/05450/05450/506033/506033.html \\
\hline John Wiley \& Sons, Inc. Publishers & http://www.wiley.com/ \\
\hline Journal of Molecular Biology & http://www.hbuk.co.uk/jmb/ \\
\hline Macmillan Publishers & http://www.mcp.com/ \\
\hline Nature & http://www.america.nature.com/ \\
\hline New Scientist & http://www.newscientist.com/ \\
\hline On-line Journals for Immunologists & http://glamdring.ucsd.edu/others/aai/journals.html \\
\hline Oxford University Press & http://www.oup.co.uk/ \\
\hline Proceedings of the National Academy of Sciences, USA & http://journals.at-home.com/PNAS/ \\
\hline Protein Science & http://www.prosci.uci.edu/ \\
\hline Science & http://www.sciencemag.org/ \\
\hline Springer-Verlag & http://www.springer.de/ \\
\hline
\end{tabular}




\section{Table 6 - Companies and suppliers.}

\begin{tabular}{|c|c|}
\hline Company & URL \\
\hline Amersham Life Science & http://www.amersham.co.uk/life/ \\
\hline Amicon & http://www.amicon.com/ \\
\hline Beckman Instruments & http://www.beckman.com/ \\
\hline $\mathrm{BDH}$ & http://www.bucc.co.uk/vbp/bdn/index.html \\
\hline Clontech & http://www.clontech.com/ \\
\hline Dako Corporation & http://www.dakousa.com/ \\
\hline Endogen, Inc. & http://www.endogen.com/ \\
\hline Eppendorf & http://www.eppendorf.com/ \\
\hline Fluka & http://www.sigma.sial.com/Fluka/ \\
\hline Glaxo Wellcome & http://www.glaxowellcome.co.uk/ \\
\hline Invitrogen & http://www.invitrogen.com/ \\
\hline Life Technologies, Inc & http://www.lifetech.com/ \\
\hline Merck \& Co., Inc & http://www.merck.com/ \\
\hline Millipore & http://www.millipore.com/ \\
\hline New England Biolabs & http://www.neb.com/ \\
\hline Novagen & http://www.novagen.com/ \\
\hline Perstorp Corporation & http://www.perstop.se/Welcome.html \\
\hline PerSeptive Biosystems & http://www.pbio.com/ \\
\hline Perkin-Elmer & http://www.perkin-elmer.com/ \\
\hline Pharmacia Biotech & http://www.biotech.pharmacia.se/ \\
\hline Poretics & http://www.semiweb.com/poretics/ \\
\hline Promega & http://www.promega.com/ \\
\hline R\&D Systems & http://www.rndsystems.com/ \\
\hline Sandoz & http://www.sandoz.com/ \\
\hline Scienceware & http://www.bel-art.com/ \\
\hline Sigma-Aldrich Corporation & http://www.sigma.sial.com/ \\
\hline Takara Biomedicals & http://www.foodsci.purdue.edu/egc/trade/takara/tak0e.htm \\
\hline Transduction Laboratories & http://translab.com/index.html \\
\hline VWR Scientific Products & http://www.vwrsp.com/ \\
\hline WWW Chemicals - Chemical manufacturers and distributors & http://www.chem.com/ \\
\hline WWW Virtual Library: Biotechnology - Companies & http://www.cato.com/interweb/cato/biotech/bio-co.html \\
\hline
\end{tabular}

\section{References}

1. Wooley JC \& Varma MN (1994). Computational biology: opportunity and challenges for the future. Basic Life Sciences, 63: 1-9.

2. Harper R (1995). World Wide Web resources for the biologist. Trends in $\mathrm{Ge}$ netics, 11: 223-228.

3. Jacobson D (1994). The World Wide Web for biologists. Protein Science, 3: 21592161.
4. Appel RD, Bairoch A \& Hochstrasser DF (1994). A new generation of information retrieval tools for biologists: the example of the ExPASy WWW server. Trends in Biochemistry, 19: 258-260.

5. Sikorski RS \& Peters R (1996). Cell and tissue culture on the web. Nature Biotechnology, 14: 656.

6. Franco GR \& Pena SDJ (1996). A directory of world wide web sites for human genetics. Brazilian Journal of Genetics, 19: 371374.
7. Brenner SE (1995). World Wide Web and molecular biology. Science, 268: 622-623.

8. Peters R \& Sikorski R (1996). Thermocycling on the web: A user's guide. Nature Biotechnology, 14: 517.

9. Goostein M (1996). Shopping for biotechnology products on the web: A user's guide. Nature Biotechnology, 14: 367. 